\title{
Ишемически-реперфузионные повреждения печени у пациентов после выполнения комбинированных резекционных вмешательств на поджелудочной железе и воротной вене
}

\author{
Е. В. Мушенко ${ }^{1}$, А. М. Тищенко ${ }^{1}$, Р. М. Смачило ${ }^{1}$, Т. В. Козлова ${ }^{2}$, Н. А. Ремнева ${ }^{2}$,
} Е. С. Проценко ${ }^{2}$ Н. Н. Гончарова ${ }^{3}$

${ }^{1}$ Институт общей и неотложной хирургии имени В. Т. Зайцева НАМН Украины, г. Харьков,

${ }^{2}$ Харьковский национальный университет имени В. Н. Каразина,

${ }^{3}$ Харьковский национальный медицинский университет

\section{Ischemic-reperfusion hepatic injuries in patients after performance of combined resections on pancreatic gland and portal vein}

\author{
E. V. Mushenko ${ }^{1}$, A. M. Tyshchenko ${ }^{1}$, R. M. Smachylo ${ }^{1}$, T. V. Kozlova ${ }^{2}$, N. A. Remnyova ${ }^{2}$, \\ E. S. Protsenko ${ }^{2}$, N. N. Honcharova ${ }^{3}$ \\ ${ }^{1}$ Zaytsev Institute of General and Urgent Surgery, Kharkov, \\ ${ }^{2}$ Karazin Kharkov National University, \\ ${ }^{3}$ Kharkov National Medical University
}

\section{Реферат}

Цель. Разработка алгоритма ранней диагностики тромбоза вен портомезентериального конфлюенса у пациентов после выполнения резекционных вмешательств на поджелудочной железе (ПЖ), сопровождавшихся резекцией либо повреждением воротной вены (BВ) и ее ветвей, для своевременной хирургической коррекции развившихся осложнений. Материалы и методы. Представлен анализ результатов хирургического лечения 269 пациентов, которым выполнили различную по объему резекцию ПЖ, из них у 15 (5,6\%) выполнены еще различные варианты резекции/реконструкции ВВ и ее ветвей, а у 6 (2,2\%) пациентов отмечено непреднамеренное повреждение вен портомезентериального конфлюенса. Ишемически-реперфузионные повреждения печени возникли у 17 больных, у 4 больных выявлен тромбоз ВВ и ее ветвей.

Результаты. На основании оценки выраженности исследованных параметров выделены три степени тяжести послеоперационной острой печеночной недостаточности (ОПН). Средняя и тяжелая степени послеоперационной ОПН требуют существенной коррекции проводимой интенсивной терапии. Из-за тромбоза ВВ и ее ветвей 3 из 4 больных умерли в раннем послеоперационном периоде. Хирургическая коррекция данного осложнения была выполнена 2 больным. Выводы. Тяжелая ОПН у данных пациентов может быть следствием тромбоза ВВ и ее ветвей, поэтому необходим постоянный мониторинг проходимости сосудов портомезентериального конфлюенса. Выявление тромбоза ВВ требует выполнения тромбэктомии с пластикой $\mathrm{BB}$, что дает основания рассчитывать на благоприятный результат лечения.

ключевые слова: резекция поджелудочной железы; резекция воротной вены; тромбоз воротной вены; острая печеночная недостаточность.

Abstract

Objective. Elaboration of the early diagnosis algorithm for thrombosis of the portomesenterial confluens veins in patients after performance of pancreatic resections, combined with resection or damage of portal vein and its branches for timely correction of complications developed.

Materials and methods. Analysis of results of surgical treatment was presented, concerning 269 patients, to whom pancreatic resection of various volume performed, of them in 15 (5.6\%) additionally various variants of resection/reconstruction of portal vein with its branches, and in $6(2.2 \%)$ patients unintentional damage of the porto-mesenterial confluens veins were noted. Ischemic-reperfusion hepatic damage have been occurred in 17 patients, in 4 patients a portal vein and its branches thrombosis was revealed.

Results. Three degrees of severity of postoperative acute hepatic insufficiency were delineated, basing on estimation of the investigated parameters. Middle and severe degrees of postoperative acute hepatic insufficiency demands essential correction of the intensive therapy conducted. Because of thrombosis of portal vein and its branches 3 of 4 patients died in early postoperative period. Surgical correction of this complication was performed in two patients.

Conclusion. Severe acute hepatic insufficiency may constitute a consequence of thrombosis of a portal vein and its branches, that is why a constant monitoring of passability of the portomesenterial confluens vessels is needed. Revealing of a portal vein thrombosis demands performance of thrombectomy with a portal vein plasty, what gives background to rely on favorable result of treatment.

Keywords: pancreatic resection; resection of portal vein; thrombosis of portal vein; acute hepatic insufficiency. 
Резекция поджелудочной железы (Пж) в настоящее время является стандартным вариантом хирургического лечения пациентов с доброкачественными и злокачественными опухолями этого органа, а также ряда пациентов с хроническим панкреатитом и панкреатическими кистами [1]. Особенности топографо-анатомического расположения ПЖ создают предпосылки к инвазии опухолей в сосудистые структуры, в первую очередь в воротную вену (BВ). Вместе с тем в последние десятилетия наличие инвазии опухолей в сосуды портомезентериального конфлюенса не является противопоказанием для выполнения резекции ПЖ, но требует удаления пораженных сосудов с различными вариантами их пластики/реконструкции [2]. Выполнение резекционных вмешательств на ПЖ относится к технически наиболее сложным операциям в абдоминальной хирургии. Развитие фиброзных изменений в парапанкреатической клетчатке при хроническом панкреатите либо наличие периопухолевых изменений без признаков инвазии в сосуды повышает риск повреждения ВВ и ее ветвей во время хирургического вмешательства и массивной кровопотери [3]. Ушивание поврежденных сосудов в подобных условиях является чрезвычайно сложной манипуляцией и зачастую существенно суживает ВВ и ее ветви с дальнейшим развитием тромбоза [4], что приводит к развитию ишемических повреждений печени, а при восстановлении воротного кровотока - реперфузионных ее повреждений. После выполнения хирургических вмешательств, связанных с необходимостью резекции/реконструкции ВВ, также развиваются ишемические и реперфузионные повреждения печени с возникновением острой печеночной недостаточности (ОПН), выраженность которых в первую очередь зависит от длительности прекращения портального кровотока. Выявление субклинических форм ОПН у пациентов, которым выполнены комбинированные резекционные вмешательства на ПЖ с резекцией/реконструкцией/пластикой сосудов портомезентериального бассейна, с целью выполнения ранней хирургической коррекции портального кровотока является чрезвычайно актуальным.

Цель исследования: разработка алгоритма ранней диагностики тромбоза вен портомезентериального конфлюенса у пациентов, которым выполнены резекционные вмешательства на ПЖ, сопровождавшиеся резекцией либо повреждением ВВ и ее ветвей, для своевременной хирургической коррекции развившихся осложнений.

\section{Материалы и методы исследования}

За период с 2013 по 2018 г. в клинике Института общей и неотложной хирургии имени В. Т. Зайцева НАМН Украины 269 пациентам были выполнены различные по объему резекционные вмешательства на ПЖ: панкреатодуоденальная резекция - 152 (56,5\%), дистальная резекция ПЖ со спленэктомией - 91 (33,8\%), дистальная резекция ПЖ с сохранением селезенки - 9 (3,3\%), срединная (корпоральная) резекция ПЖ - 5 (1,9\%), радикальная антеградная модульная резекция ПЖ - 12 (4,5\%), из них 8 - передняя, 4 - задняя. У 15 (5,6\%) из 269 оперированных пациентов выполнены различные по объему резекции/реконструкции/пластики ВВ и ее ветвей: резекция ствола ВВ с реконструкцией аутовеной - у 4 (26,7\%), резекция ствола ВВ с реконструкцией РТFE-графтом - у 3 (20\%), резекция стенки ВВ с пластикой аутовенозной заплатой - у 2 (13,3\%), резекция верхней брыжеечной вены с формированием вено-венозного анастомоза - у 1 $(6,7 \%)$, резекция стенки ВВ с ушиванием в поперечном направлении - у 4 (26,7\%), в продольном направлении - у 1 (6,7\%). Еще у 6 (2,2\%) пациентов отмечено непреднамеренное повреждение ВВ и ее ветвей во время операции, из них 4 (66,7\%) выполнено ушивание ВВ в продольном направлении, 2 (33,3\%) - ушивание ВВ в поперечном направлении. Таким образом, у 21 (7,8\%) больного резекционные операции на ПЖ сопровождались хирургическими манипуляциями на ВВ и ее ветвях. Среди этих пациентов хронический кальцифицирующий панкреатит с преимущественной локализацией патологического процесса в головке ПЖ выявлен у 3 (14,3\%), рак головки ПЖ - у 10 (47,6\%), дистальная холангиокарцинома - у 2 (9,5\%), рак тела (хвоста) ПЖ - у 6 (28,6\%). Мужчин было 10 (47,6\%), женщин - 11 (52,4\%). Возраст больных варьировал от 47 до 72 лет, средний возраст составил $(62 \pm 2,4)$ года. Среди 18 больных со злокачественными новообразованиями II стадия заболевания выявлена у 3 (16,7\%), III стадия - у $15(83,3 \%)$.

Продолжительность прекращения портального кровотока у больных, которым выполнили реконструкцию BВ аутовеной либо РТFE-графтом, варьировала от 35 до 62 мин, в среднем составляла $(47 \pm 3,5)$ мин. При пластике ВВ портомезентериального конфлюенса продолжительность прекращения портального кровотока в одном наблюдении составила 18 мин, в другом - 23 мин, при формировании вено-венозного анастомоза - 41 мин. Среди 11 пациентов, которым ушили дефект ВВ, только у 2 (18,2\%) применили пережатие ВВ и ее ветвей, средняя продолжительность прекращения портального кровотока составила 5 мин.

Ишемически-реперфузионные повреждения печени с развитием ОПН различной степени выраженности выявлены у 17 (81,0\%) из 21 больного после выполнения резекционных вмешательств на ПЖ с реконструкцией/ пластикой вен портомезентериального конфлюенса. Основными критериями ишемически-реперфузионных повреждений печени и развития ОПН были печеночная энцефалопатия, темп диуреза, метаболический ацидоз, повышение уровней аспартатаминотрансферазы (АСТ) и аланинаминотрансферазы (АЛТ), уровня креатинина в 2 раза и более по сравнению с исходными показателями, повышение уровня лактата более 4 ммоль/л, состояние гемодинамических параметров. Уровни сывороточных трансаминаз и креатинина определяли общепринятыми методами, наличие и выраженность печеночной энцефалопатии - по скорости выполнения пациентом теста связывания чисел. Кислотно-основное состояние крови исследовали автоматическим экспресс-методом на аппарате Medica EasyStat (Medica Corp., США). Уровень лактата сыворотки крови определяли с помощью аппарата Lactate Scout (EKF diagnostics, Германия). Для оценки эффективности портального кровотока проводили ультразвуковое исследование в режиме допплера с помощью ап- 
паратов Esaote My Lab Alpha (Италия) и Toshiba Aplio 400 (Япония). Тромбоз ВВ выявлен у 4 (19,1\%) из 21 пациента, которому выполнили вмешательство на венах портомезентериального бассейна: по классификации M. Yerdel и соавторов [5] II степень тромбоза отмечена у 2 (50\%) больных, III степень - у 1 (25\%), IV степень - у 1 (25\%) больного. Еще у 1 (33,3\%) из 3 пациентов, которым выполнили реконструкцию BВ PTFE-графтом, также выявлен тромбоз шунта. У 1 пациента выполнена тромбэктомия из ВВ, у 1 - тромбэктомия из воротной и верхней брыжеечной вен, у 1 - тромбэктомия из РТFЕ-графта.

\section{Результаты}

Выраженность ОПН прямо зависит от длительности ишемии печени, то есть времени прекращения портального кровотока. Еще одним фактором, обусловливающим тяжесть ОПН, является интраоперационная кровопотеря. Если непреднамеренное повреждение ВВ и ее ветвей сопровождалось массивной кровопотерей, несмотря на относительно небольшую продолжительность прекращения портального кровотока, отмечали тяжелую ОПН. В связи с необходимостью быстрой остановки кровотечения у 4 пациентов ушили ВВ в продольном направлении, что привело к развитию у них тромбоза ВВ и ее ветвей из-за значительного уменьшения диаметра вены в зоне швов. У 1 больного данное осложнение (тромбоз IV степени по классификации M. Yerdel и coавторов [5]) выявили интраоперационно. Выполнена тромбэктомия из воротной и верхней брыжеечной вен с пластикой их путем ушивания дефекта в поперечном направлении. Еще у 1 больного с тромбозом ВВ III степени по классификации M. Yerdel и соавторов [5] наличие тромба в ВВ выявили на 2-е сутки после операции. Во время выполнения повторного оперативного вмешательства отмечено значительное ишемическое повреждение печени. После тромбэктомии с пластикой ВВ ОПН прогрессировала, больной умер на 4-е сутки после операции. Тромбоз ВB II типа у 1 больного сопровождался быстрым прогрессированием ОПН, развитием синдрома полиорганной недоста- точности, что привело к смерти больного на 2-е сутки после операции, а сам тромбоз выявили на аутопсии. В 1 наблюдении тромбоз шунта ВВ выявили в 1-е сутки после операции, выполненная тромбэктомия из PTFE-графта была технически успешной, отмечен регресс ОПН. В остальных наблюдениях развитие ишемически-реперфузионных повреждений печени после выполнения резекционных вмешательств на ПЖ, сопровождавшихся хирургическими манипуляциями на венозных структурах портомезентериального конфлюенса, было связано с необходимостью временного частичного или полного прекращения портального кровотока.

На основании динамики изменений биохимических показателей крови, выраженности энцефалопатии мы выделили три степени ОПН, вызванной ишемически-реперфузионными повреждениями печени (см. маблииу).

Послеоперационная ОПН средней степени тяжести была выявлена у 8 (47,1\%) больных, которым выполнили резекцию ВВ и ее ветвей с последующей реконструкцией ВB путем протезирования (аутовенозного или РТFEграфтом) или формирования прямого анастомоза, если длительность прекращения портального кровотока превышала 35 мин. У 4 (23,5\%) больных тяжелая ОПН была связана с тромбозом ВВ и ее ветвей, из-за этого осложнения 3 больных умерли. У остальных 5 (29,4\%) больных развилась ОПН легкой степени на фоне непродолжительной ишемии печени. ОПН легкой степени протекала субклинически и не требовала существенной коррекции лечения. ОПН ІІ степени характеризовалась выраженными клиническими проявлениями, требовала коррекции нарушенной функции печени путем включения в терапию гепатопротекторов, нейропротекторов, заместительной терапии (20\% раствор альбумина). Тяжелая ОПН, развивающаяся в ранние сроки после операции, свидетельствовала о тромбозе ВВ и ее ветвей и требовала выполнения ранней хирургической коррекции.

Из 21 пациента, которому выполнили резекционные вмешательства на ПЖ с резекцией/реконструкцией/пластикой ВВ и ее ветвей, умерли 3 (14,3\%). Развитие тяже-

\begin{tabular}{|c|c|c|c|}
\hline \multicolumn{4}{|c|}{$\begin{array}{l}\text { Оценка выраженности послеоперационной ОПн у пациентов, которым выполнили рез } \\
\text { вмешательства на Пж, сопровождавшиеся хирургическими манипуляциями на ВВ и ее }\end{array}$} \\
\hline \multirow{2}{*}{ Показатель } & \multicolumn{3}{|c|}{ Степень печеночной недостаточности } \\
\hline & $\begin{array}{c}\text { I (легкая), } \\
n=5\end{array}$ & $\begin{array}{l}\text { II (средняя), } \\
\mathrm{n}=8\end{array}$ & $\begin{array}{l}\text { III (тяжелая), } \\
\text { n=4 }\end{array}$ \\
\hline $\begin{array}{l}\text { Время выполнения теста } \\
\text { связывания чисел, с }\end{array}$ & $60-90$ & $90-120$ & Более 120 \\
\hline Темп диуреза, мл/кг/ч & Менее 0,5 в течение 6 ч & Менее 0,25 в течение 6 ч & Анурия \\
\hline рН артериальной крови & $7,3-7,4$ & $7,2-7,3$ & Менее 7,2 \\
\hline Дефицит оснований & $0-7,0$ & $7,0-15,0$ & Более 15,0 \\
\hline АСТ, Ед/л & До 440 & $440-1000$ & Более 1000 \\
\hline АЛТ, Ед/л & До 400 & $400-800$ & Более 800 \\
\hline Лактат, ммоль/л & $4-8$ & $8-16$ & Более 16 \\
\hline Креатинин, мкмоль/л & $\begin{array}{c}\text { Повышение в } 2 \text { раза } \\
\text { в течение } 244\end{array}$ & $\begin{array}{c}\text { Повышение в } 3 \text { раза } \\
\text { в течение } 244\end{array}$ & $\begin{array}{l}\text { Повышение более чем } \\
\text { в } 3 \text { раза в течение } 24 \text { ч }\end{array}$ \\
\hline \multicolumn{4}{|c|}{$\begin{array}{ll}\text { Примечание. } & \text { Используя тест связывания чисел, необходимо учитывать вид проведенной анестезии при выполнении } \\
& \text { операции. Тест может применяться в послеоперационном периоде только у пациентов, имеющих стабильные } \\
\text { показатели гемодинамики и спонтанную вентиляцию. }\end{array}$} \\
\hline
\end{tabular}


лой ОПН после операции у всех больных было связано с формированием окклюзирующего тромбоза ВВ и ее ветвей. У 2 больных, умерших в ранние сроки после операции из-за быстрого прогрессирования ОПН, данное осложнение не было распознано прижизненно.

\section{Обсуждение}

В хирургии печени и органов гепатопанкреатобилиарной зоны синдром ишемии-реперфузии является одним из наиболее опасных повреждающих факторов, приводящих к развитию ОПН в раннем послеоперационном периоде. Диагностировать возникновение ишемии печени и степень повреждения печеночной ткани при реперфузии чрезвычайно сложно, поскольку оно развивается на тканевом уровне. ОПН, вызванная длительной интраоперационной ишемией печени, усугубляется после восстановления кровотока на фоне прогрессирования постперфузионных повреждений гепатоцитов.

Печень имеет двойное кровоснабжение: кровоток через ВВ составляет 70 - 80\%, через печеночную артерию - около 20 - 30\% суммарного печеночного кровотока, доставка кислорода при этом на 50\% осуществляется за счет кровотока по печеночной артерии, так как насыщение гемоглобина кислородом в вене ниже и составляет 75 - 85\%. Есть ряд причин, почему выполнение хирургических вмешательств на печени сопровождается ухудшением ее кровоснабжения. Ингаляционные анестетики снижают кровоток в ВВ; применение искусственной вентиляции легких с высоким давлением в дыхательных путях, в том числе при использовании положительного давления в конце выдоха, снижает венозный возврат к сердцу и сердечный выброс, что приводит к снижению печеночного кровотока. Прекращение портального кровотока, необходимое при выполнении резекции/реконструкции $\mathrm{BB}$ и ее ветвей, также значительно снижает печеночный кровоток. Все эти факторы, снижающие доставку кислорода, приводят к развитию гипоксии той или иной степени выраженности.

По чувствительности к ишемии ткань печени стоит на втором месте после ткани головного мозга [6], то есть обладает очень высокой чувствительностью к гипоксии.

Благодаря двойному кровоснабжению печени ее полная ишемия может развиваться только при полном прекращении кровотока. Чаще развивается частичная ишемия, обусловленная прекращением кровотока либо по печеночной артерии, либо по системе ВВ. Основной причиной нарушения кровотока по ВВ является ее тромбоз. Частичная ишемия приводит к тому, что часть клеток печени погибает, а часть остается неповрежденными (те зоны, где сохранился доступ кислорода в обычных или незначительно сниженных пределах). При прекращении кровотока по магистральным сосудам более всего выражены ишемические повреждения клеток печени в центре доли вокруг центральной венулы [6]. Поэтому глубина реперфузионных повреждений зависит от степени (полная, частичная) и длительности ишемии.

Клинические и лабораторные проявления ишемии печени, отражающие недостаточность функции печени, регистрируют уже спустя 20 мин полной ишемии. Частич- ная ишемия печени, вызванная применением интраоперационной васкулярной окклюзии, не превышающей допустимых временных рамок, также проявляется изменением лабораторных показателей, однако большая часть клеток печени остается неповрежденной и нарушение функции печени обратимо [7].

Одним из самых ранних лабораторных проявлений ишемии печени является прогрессирующее повышение уровня лактата крови выше 4 ммоль/л. Развивающийся лактат-ацидоз приводит к повреждению клеток тканей, непосредственно не связанных с местом оперативного вмешательства. Степень повышения уровня трансаминаз коррелирует со степенью повреждения печеночной ткани.

Клинически ОПН в первые часы после окончания выполнения оперативного вмешательства проявляется развитием гепаторенального синдрома (ГРС), который классифицируют на два типа по скорости развития: I тип развитие почечной недостаточности до терминальной в течение 2 нед, II тип - постепенное прогрессирование почечной недостаточности. У пациентов с ОПН, обусловленной ишемией, развивается ГРС І типа. Лабораторные и клинические характеристики ГРС зависят от степени его тяжести [8]. Для исключения других причин снижения темпа диуреза необходимо учитывать состояние гемодинамических параметров. Изменение последних может быть обусловлено некомпенсированной интраоперационной кровопотерей и гипотермией, которые приводят к развитию острой почечной недостаточности и без поражения функции печени. Поэтому подозрение на развитие ГРС возможно, если исключены эти факторы.

При выявлении тромбоза ВВ и/или ее ветвей в послеоперационном периоде следует немедленно выполнять повторное оперативное вмешательство с тромбэктомией, что дает возможность рассчитывать на обратимое течение ОПН. Поскольку обратимость ишемических повреждений печени зависит от степени нарушения кровотока в ней и времени, прошедшем до восстановления кровотока, то, чем позже будет восстановлен кровоток, тем большей будет степень реперфузионных повреждений ткани печени и процесс повреждения станет необратимым, что в свою очередь активизирует синдром системного воспалительного ответа с последующим развитием полиорганной недостаточности, приводящей к смерти пациента.

\section{Выводы}

1. После выполнения резекционных вмешательств на ПЖ, сопровождающихся резекцией/реконструкцией/ пластикой ВВ и ее ветвей, развиваются ишемически-реперфузионные повреждения печени и печеночная недостаточность, что является важным предиктором неблагоприятных результатов хирургического лечения данных больных.

2. Выраженность послеоперационной ОПН прямо зависит от продолжительности интраоперационной ишемии печени и степени кровопотери во время операции.

3. Маркерами ранней диагностики развития ОПН могут служить концентрация лактата, креатинина, АСТ, АЛТ в сыворотке крови, степень ацидоза, выраженность энцефалопатии и темп диуреза. 
4. Тяжелая ОПН у данных пациентов может быть следствием тромбоза ВВ и ее ветвей, поэтому наличие клинических признаков печеночной недостаточности и динамика изменений лабораторных данных являются основанием для выполнения ультразвукового исследования в режиме допплера с целью раннего выявления тромбоза ВВ.

5. Тромбоз ВВ является показанием к немедленному выполнению оперативного вмешательства - тромбэктомии с пластикой ВB.

6. С целью профилактики тромбоза ВВ необходимо отказаться от ее продольного ушивания в пользу поперечного, а также проводить адекватную антикоагулянтную терапию в послеоперационном периоде.

\section{Подтверждения}

\section{Финансирование}

Источником финансирования статьи являются частные лица - авторы статьи.

\section{Информация про участие авторов}

Мушенко Е .В. - написание статьи, анализ клинического материала; Тищенко А. М. - создание концепции статьи; Смачило Р. М. - анализ литературных источников; Козлова Т. В. - написание статьи, обработка материала; Ремнева Н. А. - обработка материалов лабораторных исследований; Проценко Е. С. - редактирование статьи; Гончарова Н. Н. - анализ литературных источников. Все авторы прочитали и одобрили окончательный вариант статьи.

Конфликт интересов

Авторы декларируют отсутствии конфликта интересов. Согласие на публикацию

Все авторы дали согласие на публикацию этой рукописи.

\section{References}

1. Cheung TT, Poon RTP, Chok KSH, Chan ACY, Tsang SHY, Dai WC, et al. Pancreaticoduodenectomy with vascular reconstruction for adenocarcinoma of the pancreas with borderline resectability. World J Gastroenterol. 2014 December 14; 20(46):17448-55. http://doi.org/10.3748/ wjg.v20.i46.17448.

2. Surov DA. Morfofunkcionalnoe obosnovanie rasshirennyh operativnyh vmeshatel'stv na podzheludochnoj zheleze s korrekciej portal'nogo krovotoka (jeksperimental'noe i anatomicheskoe issledovanie) [Dissertacija]. Sankt-Peterburg: Voenno-medicinskaja akademija im. S. M. Kirova; 2004. 22 p. [In Russian].

3. Kovalenko NA, Gajvoronskij IV, Kotiv BN, Tjagun VS, Novickaja NJu. Variantnaja anatomija vorotnoj veny $\mathrm{i}$ ee prikladnoe znachenie $\mathrm{v}$ hirurgii. Medline.ru, hirurgija 2018 mart 6;19:182-208. [In Russian].

4. Hicks CW, Glebova NO, Piazza KM, Orion K, Pierorazio PM, Lum YW, et al. Risk of venous thromboembolic events following inferior vena cava resection and reconstruction. J Vasc Surg. 2016 Apr;63(4):1004 10. http://doi.org/10.1016/j.jvs.2015.09.020.

5. Yerdel MA, Gunson B, Mirza D, Karayalçin K, Olliff S, Buckels J, et al. Portal vein thrombosis in adults undergoing liver transplantation: risk factors, screening, management, and outcome. Transplantation. 2000 May 15;69(9):1873-81. PMID: 10830225.

6. Abuelo JG. Normotensive ischemic acute renal failure. N Engl J Med. 2007 August 23;357:797-805. http://doi.org/10.1056/NEJMra 064398.

7. Abu-Amara M, Gurusamy K, Hori S, Glantzounis G, Fuller1B, Davidson BR. Systematic review of randomized controlled trials of pharmacological interventions to reduce ischaemia-reperfusion injury in elective liver resection with vascular occlusion. HPB (Oxford). $2010 \mathrm{Feb}$;2(1):414. http://doi.org/10.1111/j.1477-2574.2009.00120.x.

8. Rahbari NN, Garden OJ, Padburi R, Brooke-Smith M, Crawford M, Adam R, et al. Posthepatectomy liver failure: a definition and grading by the International Study Group of Liver Surgery (ISGLS). Surgery, 2011 May;149(5):713-24. http://doi.org/10.1016/j.surg.2010.10.001

Поступила 29.11.2018 